19

\title{
Отражение света от плазмонных пленок золота и серебра: сравнение эксперимента и теории
}

\author{
(C) В.Ф. Аскирка ${ }^{1}$, Д.В. Гузатов ${ }^{1}$, С.А. Маскевич ${ }^{1,2}$ \\ ${ }^{1}$ Гродненский государственный университет им. Янки Купалы, \\ 230023 Гродно, Республика Беларусь \\ ${ }^{2}$ Международный государственный экологический институт им. А.Д. Сахарова \\ Белорусского государственного университета, \\ 220070 Минск, Республика Беларусь \\ e-mail: vaskirka@tut.by
}

Поступила в редакцию 09.07.2020 г.

В окончательной редакции 28.09.2020 г.

Принята к публикации 05.10.2020 г.

\begin{abstract}
Исследованы спектры отражения света плазмонными металлическими пленками, напыленными в вакууме на диэлектрические подложки и отожженными впоследствии при высокой температуре. Для объяснения оптических свойств плазмонных пленок используется теоретическая модель плоскослоистой металлодиэлектрической среды. Один из слоев такой структуры имеет диэлектрическую проницаемость исследуемого металла, а второй слой - диэлектрическую проницаемость, вычисляемую в рамках приближения МаксвеллаГарнетта для гетерогенной среды, состоящей из взвеси металлических наноэллипсоидов в воздухе. Введение данного слоя позволяет качественно учесть неоднородности поверхности плазмонной пленки. Показано, что разработанная модель позволяет объяснить ряд особенностей в экспериментальных спектрах отражения.
\end{abstract}

Ключевые слова: плазмонные пленки, спектры зеркального отражения, модель эффективной среды, приближение Максвелла-Гарнетта.

DOI: $10.21883 /$ OS.2021.02.50562.195-20

\section{Введение}

Поверхности наноструктурированных металлических пленок позволяют эффективно концентрировать падающее электромагнитное поле в объемах размерами много меньшими дифракционного предела благодаря возбуждению поверхностных локализованных и(или) распространяющихся плазмонов с большим, чем у света, волновым вектором. При взаимодействии света с плазмонами происходит значительное усиление интенсивности поля, увеличение плотности фотонных состояний [1], что приводит к усилению ряда оптических эффектов, находящих широкое практическое применение [2-8].

В силу естественных ограничений возможностей пространственного разрешения оптических методов в субнанометровом масштабе моделирование свойств нанокомпозитных сред (включая плазмонные пленки благородных металлов) является важным этапом понимания физических процессов в них. Различные теории эффективной среды применимы к большому числу дисперсных сред для описания их оптических характеристик [9], например, теории Шенга, Хашин-Штрикмана, Бергмана, Винера, а также наиболее универсальные и потому наиболее популярные теории Бруггемана и МаксвеллаГарнетта $[10,11]$. В основе теорий лежит определение комплексной диэлектрической проницаемости нанокомпозитной среды через диэлектрические проницаемости составляющих ее компонентов или наоборот. Характерной особенностью плазмонных металлических пле- нок (ПМП) является наличие оптического резонанса в видимой области в отличие от гладких и сплошных (зеркальных) металлических пленок с неселективным отражением в видимой длинноволновой и инфракрасной области. Плазмонная полоса формируется откликом взаимодействующих металлических частиц пленки.

Теория Максвелла-Гарнетта [12] изначально была разработана исходя из предположения о сферичности металлических частиц и пренебрежения взаимодействием между ними, что достигается при малых значениях фактора объемного заполнения. Размеры частиц при этом много меньше длины волны падающего света. В случае ПМП чаще всего рассматриваются частицы в виде сфероидов одинаковой формы, т.е. с одинаковым отношением полуосей сфероидов, но разных размеров, которые остаются при этом по-прежнему значительно меньше длины волны света [11]. Напыленные в вакууме ПМП (особенно после их термического отжига) можно представить в виде ансамбля наночастиц, самоорганизованных поверх сплошной металлической поверхности [13]. Ранее было показано [14-20], что расширенная теория Максвелла-Гарнетта вполне приемлема для соотнесения эффективной диэлектрической функции с подобной морфологией поверхности. Таким образом, анализ возможностей и ограничений разных моделей приводит к выбору расширенной теории Маквелла-Гарнета для моделирования процессов отражения света плазмонными металлическими пленками. Целью представленной работы является теоретическое моделирование зеркаль- 

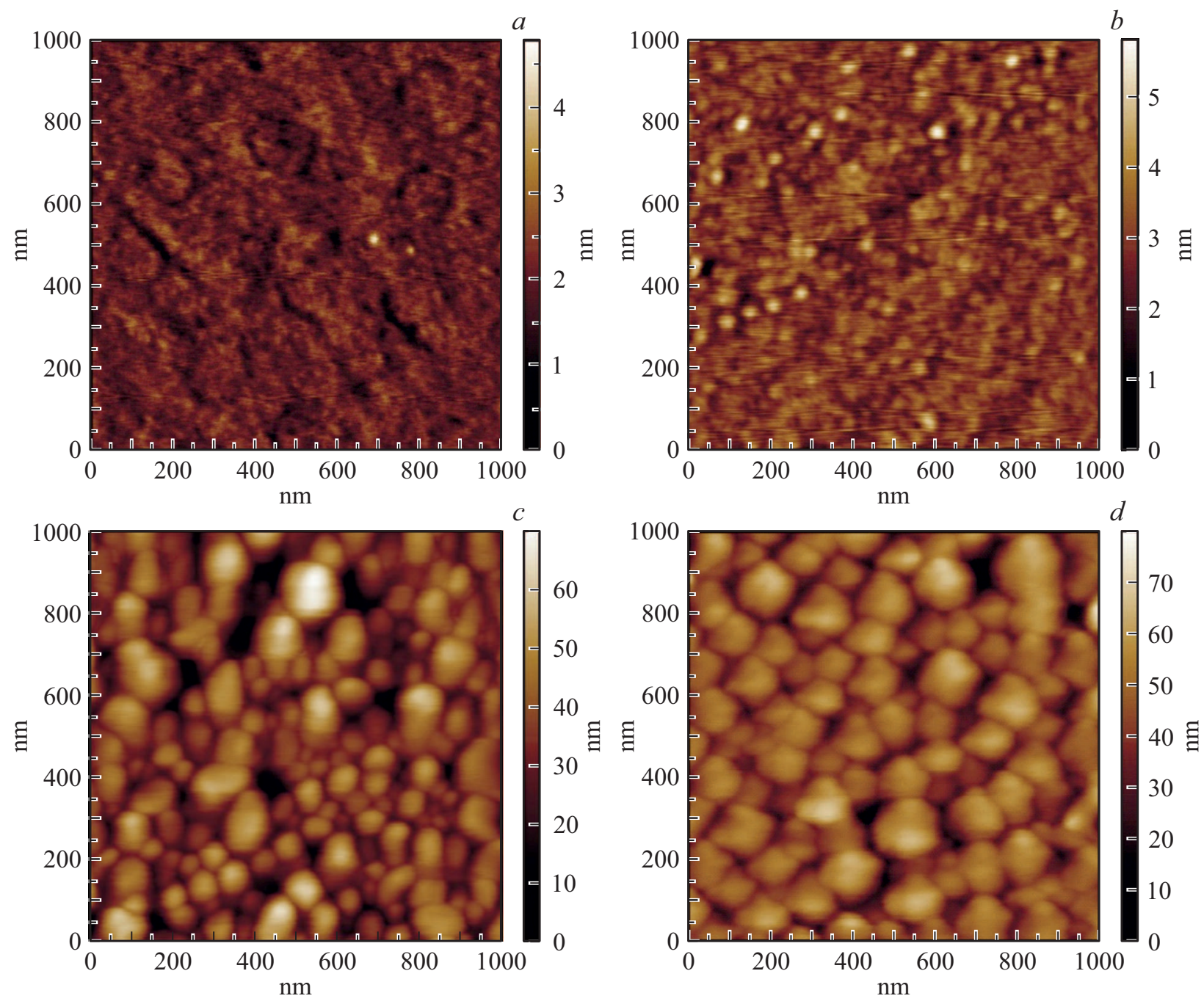

Рис. 1. АСМ-изображения плазмонных пленок: ППЗ $(a, c)$, ППС $(b, d)$, напыленные $(a, b)$ и отожженные при $340-350^{\circ} \mathrm{C}(c, d)$.

ного отражения ПМП, согласованное с экспериментальными данными, для лучшего понимания закономерностей взаимосвязи морфологии наноструктурированной металлической поверхности с ее оптическим откликом в дальнее поле.

\section{Эксперимент}

Плазмонные пленки золота (ППЗ) и плазмонные пленки серебра (ППС) были получены методом вакуумного напыления металла на кварцевые подложки по оригинальным методикам лаборатории молекулярной спектроскопии и оптики наноструктур ГрГУ имени Янки Купалы [21,22]. Термический отжиг пленок, осажденных на кварцевые подложки, производился в термостатированной муфельной печи на воздухе в несколько этапов при различных температурах. Время отжига на каждом из этапов подбиралось индивидуально по отношению к отдельным образцам. После каждой стадии отжига пленки охлаждались до комнатной температуры, после чего записывались спектры их зеркального отражения, а также АСМ-изображения поверхности (рис. 1).

Сравнение геометрических размеров исходной (напыленной в вакууме, неотожженной) и отожженной пленок, топографии которых зарегистрированы с помощью АСМ-технологии (рис. 1), позволяет сделать вывод, что пленка неотожженная представляет собой рыхлую сплошную пленку с разрывами и наноразмерными шероховатостями на поверхности. Сплошная часть пленки, непосредственно осажденная на кварцевую или слюдяную подложку, является аморфной [23]. В процессе отжига формируются и растут наноструктуры, сложную форму которых можно представлять ансамблями отдельных и перекрывающихся эллипсоидов различных размеров и форм, а толщина сплошной части пленки при этом уменьшается, в некоторых местах вплоть до разрыва пленки до диэлектрической подложки. В итоге получается серебряная или золотая пленка островкового типа с высокой степенью кристалличности [23]. В зависимости от массовой толщины осажденного на 
подложку металла островки (эллипсоидальной формы) соприкасаются или не соприкасаются друг с другом. Как видно из рис. $1, c, d$, получаемые таким образом ПМП имеют широкий спектр размеров как в латеральной плоскости, так и по высоте. Отожженные ППЗ и ППС являются объектами исследования в данной работе.

Для получения золотых и серебряных зеркал процесс вакуумного напыления металла на кварцевые подложки повторялся несколько раз с соблюдением отработанных режимов до состояния, при котором образцы становятся оптически непрозрачными.

Спектры отражения ППЗ, ППС и зеркал регистрировались с использованием спектрофотометра Specord 200 (Carl Zeiss, Йена, Германия) и входящей в комплект оборудования отражательной приставки (диапазон варьирования углов падения 10-60). АСМ-изображения поверхностей металлических пленок были получены с использованием АCM-микроскопа „NT-MDT NTEGRA“ (Москва, Россия). Сканирование поверхности осуществлялось в режиме постоянной силы с использованием полуконтактного метода.

\section{Теория}

Для описания отражения света ПМП рассмотрим структуру из двух прилегающих плоскопараллельных слоев с толщинами $h_{1}$ и $h_{2}$, лежащих на диэлектрической подложке (рис. 2). Верхнее полупространство $(z>0)$ пусть будет заполнено веществом с единичной диэлектрической проницаемостью (воздух); нижнее полупространство расположено в области отрицательных $z$ и пусть оно будет заполнено диэлектриком с проницаемостью $\varepsilon_{d}$. Двухслойная структура находится в области $0>z>-\left(h_{1}+h_{2}\right)$, а плоскости $z=0, z=-h_{1}$ и $z=-\left(h_{1}+h_{2}\right)$ - задают поверхности раздела сред. Первый слой (индекс „1“), расположенный в области $0>z>-h_{1}$ будет описывать неоднородности в виде шероховатостей металлической пленки (см. далее). Второй слой (индекс „2“), расположенный в области $h_{1}>z>-h_{2}$, описывает металлическую пленку без шероховатостей. Диэлектрические проницаемости первого и второго слоев $\varepsilon_{1}$ и $\varepsilon_{2}$ соответственно.

Рассмотрим падение из верхнего полупространства на плоскослоистую структуру эллиптически поляризованной электромагнитной волны, имеющей напряженности электрического $\left(\mathbf{E}_{0}\right)$ и магнитного $\left(\mathbf{H}_{0}\right)$ полей в следующем виде (множитель зависимости от времени $\exp (-i \omega t)$ - опущен):

$$
\begin{gathered}
\mathbf{E}_{0}=\left(A_{0} \mathbf{e}_{x}-B_{0} \cos \theta_{0} \mathbf{e}_{y}-B_{0} \sin \theta_{0} \mathbf{e}_{z}\right) \\
\times \exp \left(i k_{0} y \sin \theta_{0}-i k_{0} z \cos \theta_{0}\right), \\
\mathbf{H}_{0}=-\frac{i}{k_{0}} \operatorname{rot} \mathbf{E}_{0},
\end{gathered}
$$

где $A_{0}$ и $B_{0}$ - амплитуды падающей волны, задающие ее поляризацию: $B_{0}=0$ соответствует волне $s$-поляризации, а $A_{0}=0$ соответствует волне $p$-поляризации;

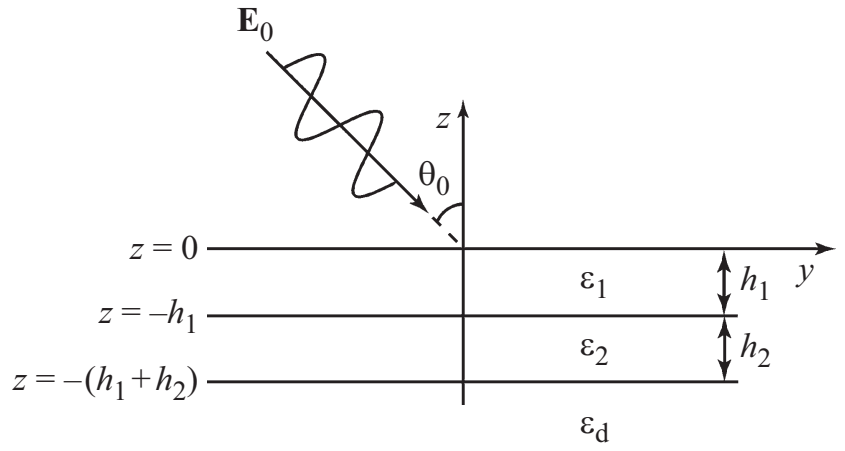

Pис. 2. Геометрия задачи о плоскослоистой структуре.

$\mathbf{e}_{x}, \mathbf{e}_{y}$ и $\mathbf{e}_{z}-$ орты декартовой системы координат вдоль соответствующих осей; $0 \leq \theta_{0} \leq \pi / 2-$ угол, который составляет вектор, указывающий направление распространения волны, с отрицательным направлением оси $z$ декартовой системы координат (рис. 2); $k_{0}-$ волновое число в вакууме.

Падающая волна (1) порождает волну, отраженную от плоскослоистой структуры, распространяющуюся внутри структуры, и волну, прошедшую через структуру в нижнее полупространство. Подобная задача нахождения индуцированных волн многократно решалась (например, [24,25]), поэтому далее мы не будем приводить способ нахождения полей и промежуточные выражения, а сразу приведем выражения для коэффициента отражения $R$. Итак, в результате вычислений можно получить:

$$
R=\frac{\left|A_{u}\right|^{2}+\left|B_{u}\right|^{2}}{\left|A_{0}\right|^{2}+\left|B_{0}\right|^{2}},
$$

где $A_{u}$ и $B_{u}$ - амплитуды отраженной волны, для которых можно найти выражения:

$$
\begin{aligned}
& a_{u 1}^{s}+a_{12}^{s} \exp \left(2 i \beta_{1} h_{1}\right)+a_{u 1}^{s} a_{12}^{s} a_{2 d}^{s} \exp \left(2 i \beta_{2} h_{2}\right) \\
& A_{u}=A_{0} \frac{+a_{2 d}^{s} \exp \left[2 i\left(\beta_{1} h_{1}+\beta_{2} h_{2}\right)\right]}{1+a_{u 1}^{s} a_{12}^{s} \exp \left(2 i \beta_{1} h_{1}\right)+a_{12}^{s} a_{2 d}^{s} \exp \left(2 i \beta_{2} h_{2}\right)} \text {, } \\
& +a_{u 1}^{s} a_{2 d}^{s} \exp \left[2 i\left(\beta_{1} h_{1}+\beta_{2} h_{2}\right)\right] \\
& a_{u 1}^{p}+a_{12}^{p} \exp \left(2 i \beta_{1} h_{1}\right)+a_{u 1}^{p} a_{12}^{p} a_{2 d}^{p} \exp \left(2 i \beta_{2} h_{2}\right) \\
& B_{u}=B_{0} \frac{+a_{2 d}^{p} \exp \left[2 i\left(\beta_{1} h_{1}+\beta_{2} h_{2}\right)\right]}{1+a_{u 1}^{p} a_{12}^{p} \exp \left(2 i \beta_{1} h_{1}\right)+a_{12}^{p} a_{2 d}^{p} \exp \left(2 i \beta_{2} h_{2}\right)} \text {, } \\
& +a_{u 1}^{p} a_{2 d}^{p} \exp \left[2 i\left(\beta_{1} h_{1}+\beta_{2} h_{2}\right)\right]
\end{aligned}
$$

в которых обозначены коэффициенты

$$
\begin{gathered}
a_{u 1}^{s}=\frac{k_{0} \cos \theta_{0}-\beta_{1}}{k_{0} \cos \theta_{0}+\beta_{1}}, a_{12}^{s}=\frac{\beta_{1}-\beta_{2}}{\beta_{1}+\beta_{2}}, a_{12}^{s}=\frac{\beta_{2}-\beta_{d}}{\beta_{2}+\beta_{d}}, \\
a_{u 1}^{p}=\frac{\varepsilon_{1} k_{0} \cos \theta_{0}-\beta_{1}}{\varepsilon_{1} k_{0} \cos \theta_{0}+\beta_{1}}, a_{12}^{p}=\frac{\varepsilon_{2} \beta_{1}-\varepsilon_{1} \beta_{2}}{\varepsilon_{2} \beta_{1}+\varepsilon_{1} \beta_{2}}, \\
a_{2 d}^{p}=\frac{\varepsilon_{d} \beta_{1}-\varepsilon_{2} \beta_{d}}{\varepsilon_{d} \beta_{1}+\varepsilon_{2} \beta_{d}},
\end{gathered}
$$

где $\beta_{n}=k_{0} \sqrt{\varepsilon_{n}-\sin ^{2} \theta_{0}}-$ продольное волновое число волны в $n$-м слое $(n=1,2) ; \beta_{d}=k_{0} \sqrt{\varepsilon_{d}-\sin ^{2} \theta_{0}}-$ 
продольное волновое число прошедшей в подложку волны.

В работе мы используем модель, в соответствии с которой диэлектрическая проницаемость $\varepsilon_{1}$ является некоторой эффективной диэлектрической проницаемостью, соответствующей гетерогенной среде, образованной малыми по сравнению с длиной волны трехосными эллипсоидами (наноэллипсоидами) из материала с диэлектрической проницаемостью $\varepsilon_{2}$, взвешенными в воздухе. Такая модель позволяет приближенно учесть неоднородности и шероховатости, возникающие на поверхности слоя 2. Мы будем рассматривать случай произвольно ориентированных наноэллипсоидов (случай изотропного слоя 1), имеющих одинаковые соотношения между соответствующими полуосями, но при этом не обязательно имеющими одинаковый размер. В этом случае для эффективной диэлектрической проницаемости можно получить выражение [26]:

$$
\begin{aligned}
\varepsilon_{1}= & 1+\frac{\eta}{3}\left(\sum_{j=x, y, z} \frac{\varepsilon_{2}-1}{1+N_{j}\left(\varepsilon_{2}-1\right)}\right) / \\
& {\left[1-\frac{\eta}{3} \sum_{j=x, y, z} \frac{N_{j}\left(\varepsilon_{2}-1\right)}{1+N_{j}\left(\varepsilon_{2}-1\right)}\right], }
\end{aligned}
$$

где $\eta$ - параметр заполнения, который в случае одинаковых наночастиц вычисляется как объем наночастицы, умноженный на их концентрацию;

$$
N_{j}=\frac{a_{x} a_{y} a_{z}}{2} \int_{0}^{\infty} \frac{d u}{\left(u+a_{j}^{2}\right) \sqrt{\left(u+a_{x}^{2}\right)\left(u+a_{y}^{2}\right)\left(u+a_{z}^{2}\right)}}
$$

- фактор деполяризации наноэллипсоида; $a_{x}, a_{y}$ и $a_{z}$ - полуоси наноэллипсоида вдоль соответствующих декартовых осей. В частном случае $a_{x}=a_{y}=a_{z}$ из (5) получается выражение для эффективной диэлектрической проницаемости гетерогенной среды, образованной взвешенными в воздухе наносферами, впервые полученное Максвеллом-Гарнеттом [12]. Заметим, что теория Максвелла-Гарнетта, а также другие аналогичные ей теории [11,27], применяемые для вычисления эффективной диэлектрической проницаемости гетерогенных сред, широко используются для описания свойств различных метаматериалов [28-30], лазерных сред, представляющих собой активную среду с металлическими наночастицами [31,32], оптических свойств морской пены [33,34], в эллипсометрических исследованиях толщины слоя наносимого материала [16,17].

\section{Результаты и обсуждение}

\section{Золотые и серебряные зеркала}

Для использования описанной выше задачи о плоскослоистой структуре в моделировании спектров зеркального отражения ПМП необходимо выяснить предварительно применимость подхода к описанию отражательных свойств плоских металлических зеркал. В этом случае нет необходимости учитывать слой эффективной среды (достаточно положить его толщину $h_{1}=0$ ) и рассматривать плоский слой золота или серебра (толщиной $h_{2}$ ). АСМ-изображения золотых и серебряных зеркал, полученных вакуумным напылением в нашей лаборатории, демонстрируют наличие наноструктурированной поверхности с наночастицами высотой в основном до десятка нанометров. Причем это поверхность достаточно толстой проводящей пленки со свойствами объемного металла. Так как длина волны падающего излучения во всем оптическом диапазоне значительно превосходит размеры „неровностей“, то экспериментально полученные зеркала вполне можно рассматривать как плоские слоистые структуры. Это позволяет сравнивать оптические характеристики образцов, полученные экспериментально и в рамках рассматриваемой теоретической модели. Рассмотрим их на примере золотых образцов, так как общие выводы и для золотых, и для серебряных зеркал одинаковые.

На рис. 3 представлены экспериментальные $(a)$ и расчетные $(b)$ спектры отражения золотых зеркал для $s$ - и $p$-поляризованного света при различных углах его падения.

Расчет спектров отражения зеркал в модели определяется лишь толщиной плоского сплошного слоя металла. Толщина слоя составляла $h_{2}=100 \mathrm{~nm}$, хотя изменений в спектрах не наблюдается, начиная с толщины $60-70 \mathrm{~nm}$, то есть уже при таких размерах сплошной пленки ее можно считать „толстой“ и зеркальной.

В целом расчетные спектры отражения повторяют экспериментальные зависимости и для золотых, и для серебряных зеркал и состояний поляризации падающего излучения. Особенно сходство выражено для золотых образцов, спектры которых демонстрируют наличие широкой области неселективного отражения от $500 \mathrm{~nm}$. Для серебряных зеркал эта область начинается от $320 \mathrm{~nm}$, однако экспериментальные спектры содержат полосу просветления около $400 \mathrm{~nm}$, что не в полной мере отражается в модельных спектрах. Для выяснения возможного происхождения этого спектрального „провала“ в силу значительной реакционной способности серебряной поверхности, было принято допущение о наличии слоя оксида серебра (в частности, $\mathrm{Ag}_{2} \mathrm{O}$ как наиболее термостабильной фазы [35]). Значения диэлектрических констант оксида [16] позволили ввести в теоретическую модель слой эффективной среды $\left(h_{1}\right)$. Расчеты показали наличие отражательной особенности около $400 \mathrm{~nm}$ спектре. Таким образом, можно утверждать, что наличие указанной области просветления обусловлено окислением поверхности серебряного зеркала.

Общий характер поведения экспериментальных и теоретических спектров отражения зеркал при использовании поляризованного падающего излучения согласуется: во всем рассматриваемом диапазоне длин волн с увеличением угла падения при $s$-поляризации отражательная способность пленок увеличивается, а при p-поляризации - уменьшается (рис. 3). 

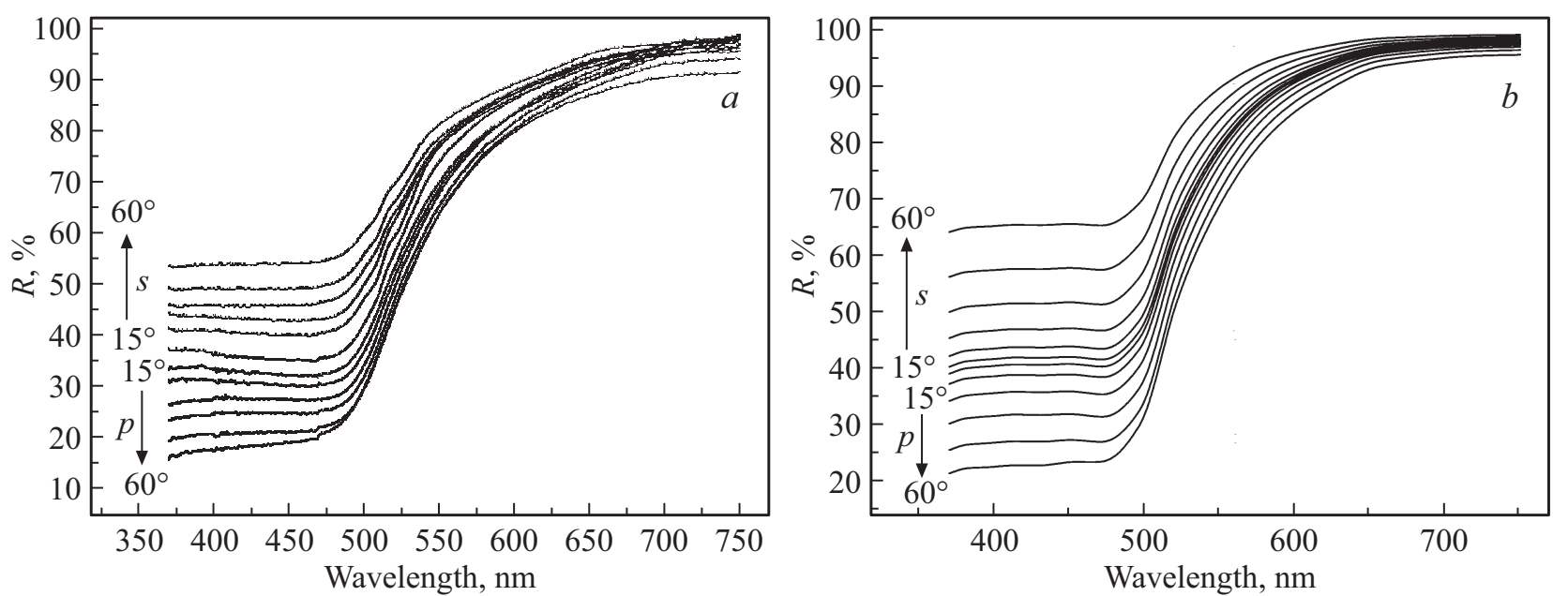

Pис. 3. Спектры зеркального отражения золотого зеркала на кварцевой подложке: измеренные $(a)$ и рассчитанные $(b)$ для $s$ - и $p$-поляризованного света при различных углах падения света.

Таким образом, предложенная модель плоскослоистой структуры показывает свою пригодность для расчетов отражения падающего электромагнитного излучения зеркальными поверхностями серебра и золота.

\section{Плазмонные пленки золота и серебра}

В отличие от зеркал, ПМП представляют собой развитую структуру, находящуюся в контакте с диэлектрической средой, с наномасштабными частицами, распределенными в широком диапазоне размеров, как в латеральной плоскости, так и по высоте [21,22]. В частности, высота металлических частиц может составлять от нескольких нанометров до нескольких десятков нанометров [36], а характер их распределения по размерам и форме обусловлен особенностями технологического приготовления образцов [22]. Интегральный оптический отклик различных массивов подобных частиц также различный, что и регистрируется в спектрах отражения.

На рис. 4 представлены спектры зеркального отражения ППЗ $(a)$ и ППС $(b)$ для $s$ - и р-поляризованного света при различных углах падения. В отичие от спектров отражения зеркал, в ПМП наблюдается селективность в отражении, обусловленная коллективным откликом наноразмерных плазмонных металлических частиц. Так, для ППЗ имеется одна плазмонная полоса с максимумом около $590 \mathrm{~nm}$ (рис. 4, $a$ ), для ППС присутствуют коротковолновая и длинноволновая плазмонные полосы около 345 и $445 \mathrm{~nm}$ соответственно (рис. 4, b). Кроме зеркального отражения падающее излучение испытывает и диффузное рассеяние, вклад которого не превышает $10 \%$ [22], поэтому им можно пренебречь.

Для моделирования отражательных свойств ПМП используем задачу плоскослоистой структуры. В этой модели первый слой толщиной $h_{1}-$ слой эффективной среды, прилегающий к нему второй слой толщиной $h_{2}$ - сплошной слой металла. Второй слой находится в контакте с диэлектрической подложкой (в нашем случае пластинка кварца) полубесконечной толщины. Таким образом, оптический отклик слоистой системы будет определяться значениями диэлектрических констант золота и серебра [37], а также изменяющимися параметрами: толщинами слоев $h_{1}, h_{2}$, параметром заполнения $\eta$ и соотношением между полуосями наноэллипсоидов.

Предварительная оценка значимости расхождения экспериментальных и теоретически рассчитанных спектров отражения производилась с использованием критерия $\chi^{2}$, после чего подбирались значения изменяющихся параметров до достижения соответствия по двум параметрам: положения плазмонных максимумов и абсолютного значения коэффициентов отражения в них.

Процесс подбора параметров представим на примере ППЗ и ППС для $s$-поляризованного света при угле падения $20^{\circ}$. На рис. 5, а представлена серия расчетных спектров отражения ППЗ с фиксированными значениями толщин слоя гетерогенной среды $h_{1}=10 \mathrm{~nm}$, металла $h_{2}=2 \mathrm{~nm}$, параметра заполнения $\eta=0.35$ при изменении соотношения между осями эллипсоидов от $3: 5: 5$ до $3: 5: 13$. Для сравнения здесь же приведен экспериментальный спектр, а также расчетный для включений в виде сфероидов (при соотношении $3: 3: 3$ ). С увеличением вытянутости эллипсоидов плазмонная полоса испытывает значительный батохромный сдвиг при одновременном увеличении отражающей способности. Полуширина полосы при этом изменяется в пределах от 32.7 до $55.8 \mathrm{~nm}$. Несмотря на значительное отличие от полуширины экспериментального спектра $(206 \mathrm{~nm})$, максимум плазмонной полосы модельного спектра при соотношении 3:5:10 совпадает с ним по положению и абсолютному значению коэффициента отражения.

На рис. 5, $b$ представлено семейство расчетных спектров отражения ППС с фиксированными значениями толщин слоя гетерогенной среды $h_{1}=24 \mathrm{~nm}$, металла $h_{2}=2 \mathrm{~nm}$, параметра заполнения $\eta=0.15$ при измене- 

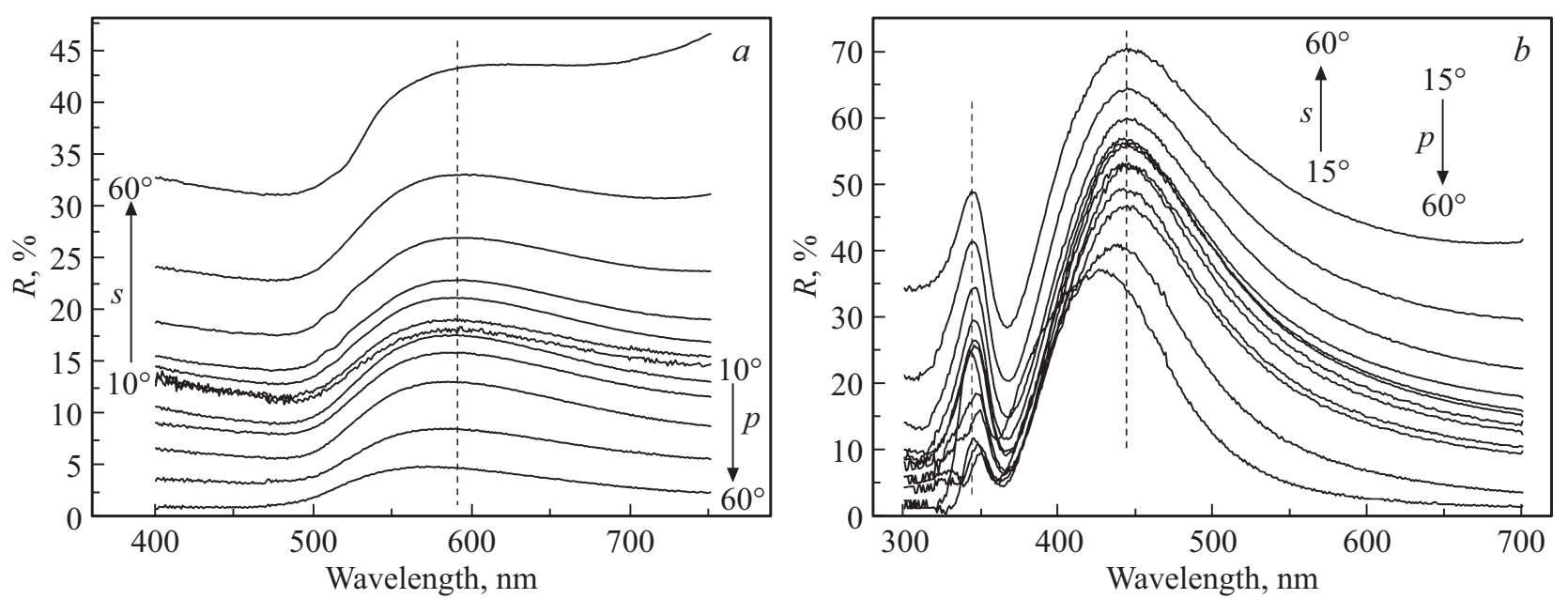

Pис. 4. Спектры отражения ППЗ $(a)$ и ППС $(b)$ на кварцевой подложке для $s$ - и $p$-поляризованного излучения от углов падения.
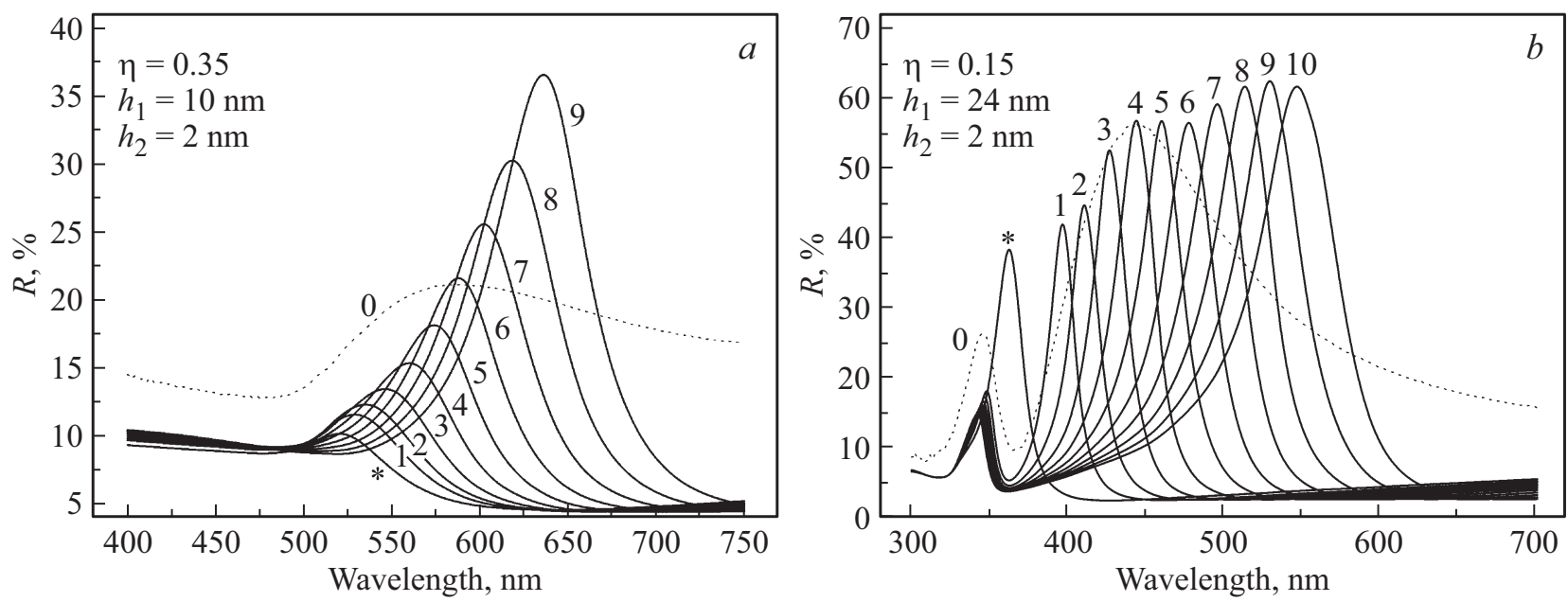

Рис. 5. Модельные спектры отражения ППЗ $(a)$ и ППС $(b)$ для $s$-поляризованного излучения при угле падения $20^{\circ}$ при изменении соотношений между полуосями эллипсоидов: (a) 0 - экспериментальный спектр, $1-9$ соответствуют $3: 5: 5-3: 5: 13$, * - расчетный спектр для случая сфероидов $(3: 3: 3)$; (b) 0 - экспериментальный спектр, $1-10$ соответствуют 4:4:7-4:4:16,

* - расчетный спектр для случая сфероидов $(4: 4: 4)$.

нии соотношения между осями эллипсоидов от $4: 4: 7$ до $4: 4: 16$. Здесь для сравнения также приведен экспериментальный спектр и расчетный для включений в форме сфероидов (при соотношении 4:4:4). Прежде всего обращает на себя внимание факт отсутствия двух плазмонных полос при моделировании сферическими включениями, что исключает использование такого подхода для моделирования спектров отражения ППС. Очевидно, что фактор формы играет в этом случае ключевую роль, так как для сферических включений наблюдается вырождение плазмонных резонансов [38], приводящее к регистрации лишь одной плазмонной полосы (рис. 5, b). Рассчитанная плазмонная полоса испытывает значительный длинноволновый сдвиг при увеличении вытянутости эллипсоидов как в случае золотых, так и серебряных гетерогенных сред. Однако при этом увеличение отражающей способности для ППС не является таким значительным, как для золотых образцов. Одновременно со спектральным сдвигом возрастает полуширина длинноволновой полосы от 18.8 до $57.2 \mathrm{~nm}$. Отметим, что эта величина значительно меньше полуширины соответствующей резонансной полосы экспериментального спектра $(126 \mathrm{~nm})$. Тем не менее максимум плазмонной полосы модельного спектра при соотношении 4:4:10 совпадает с ним по положению и абсолютному значению коэффициента отражения.

Для ППЗ и ППС проводилась подгонка параметров модели следующим образом: 1) при фиксированных толщинах слоёв $\left(h_{1}\right.$ и $\left.h_{2}\right)$, соотношения между полуосями эллипсоидов и изменяющимся параметром заполнения $\eta ; 2$ ) при фиксированных толщине слоя $h_{2}$, соотношения между полуосями эллипсоидов, параметром заполнения и изменяющимся значением толщины слоя эффективной среды $h_{1}$. 

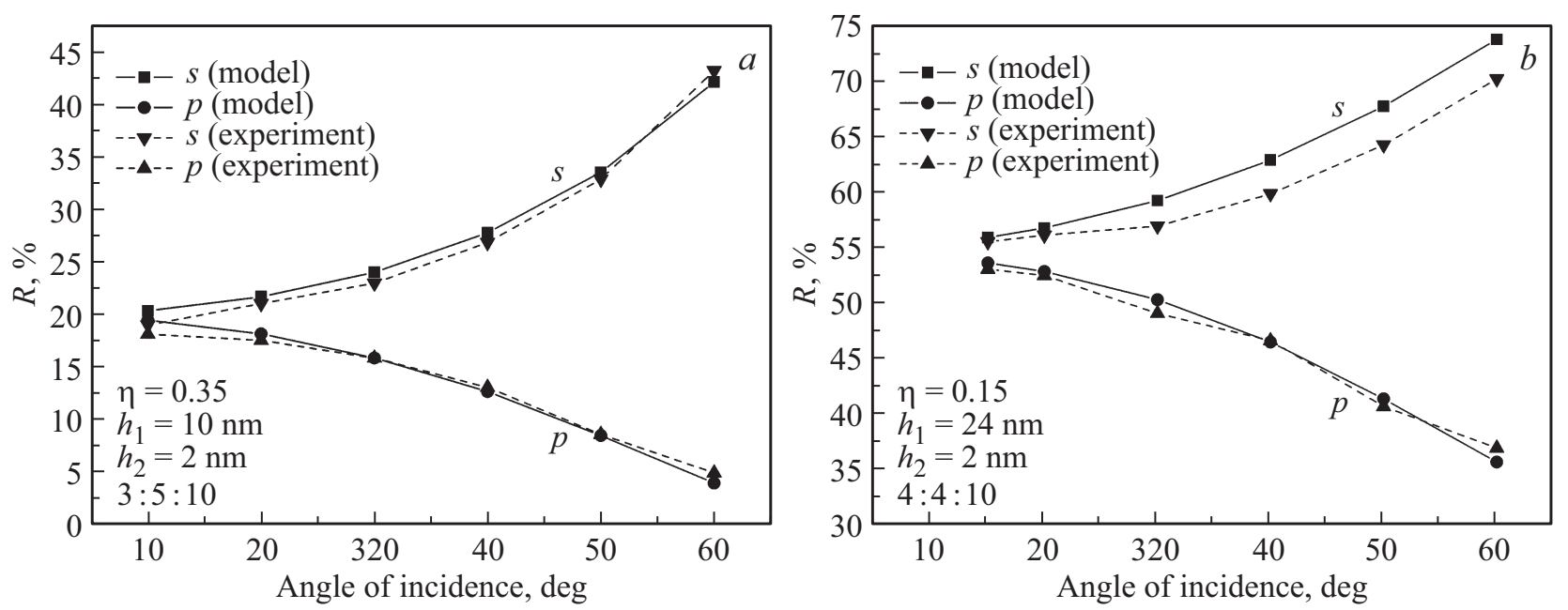

Рис. 6. Расчетные и измеренные зависимости коэффициентов отражения $s$ - и $p$-поляризованного излучения в максимуме плазмонных полос от углов падения для ППЗ (около $590 \mathrm{~nm}$ ) $(a)$ и ППС (около $445 \mathrm{~nm})(b)$.

Прежде всего модель „чувствительна“ к наличию гетерослоя, так как в случае его отсутствия $(\eta=0)$ плазмонные полосы не наблюдаются в принципе. При увеличении значения параметра заполнения $\eta$ фиксируется увеличение отражающей способности слоистой структуры с незначительным сдвигом максимума плазмонных полос в длинноволновую область и для ППЗ, и для ППС. При увеличении толщины слоя эффективной среды $h_{1}$ (при прочих неизменных параметрах) для обоих видов ПМП положение плазмонных полос не изменяется. При этом коэффициент отражения в максимуме увеличивается.

При использовании модели для $p$-поляризованного света наблюдается схожее поведение расчетных спектров, как и для $s$-поляризованного света.

Таким образом, согласно выбранным параметрам (положение максимума $\lambda^{\max }$ плазмонной полосы спектра отражения и коэффициента отражения при $\lambda^{\mathrm{max}}$ ) оптимальными в модели для ППЗ являются значения $h_{1}=10 \mathrm{~nm}$, $h_{2}=2 \mathrm{~nm}, 3: 5: 10, \eta=0.35$, для ППС $-h_{1}=24 \mathrm{~nm}$, $h_{2}=2 \mathrm{~nm}, 4: 4: 10, \eta=0.15$. Для ППС расчетный и экспериментальный спектры совпадают по положению обеих плазмонных полос и значению коэффициента отражения в максимуме длинноволновой полосы. Важно также отметить, что для оптимальных параметров модели толщина гетерослоя $h_{1}$ отражает базовое отличие реальных ППЗ и ППС: согласно данным АСМ, средний размер плазмонных частиц в пленках серебра в $2-3$ раза больше, чем в пленках золота.

В соответствии с моделью гетерогенная среда образуется произвольно ориентированными трехосными наноэллипсоидами из золота или серебра, взвешенными в воздухе. При расчетах фиксируется соотношение между их полуосями, что означает единообразие форм частиц. Размер частиц различен, и его оценку можно произвести по величине слоя $h_{1}$. В силу распределения форм и размеров плазмонных частиц пленки в широком диапазоне параметров экспериментальные плазмонные полосы имеют многомодовую природу, что явно демонстрирует рис. 5. Модель, используемая в работе, не чувствительна к разнообразию мод, так как использует лишь одно из соотношений между полуосями эллипсоидов. Изменение этого соотношения позволяет получать отдельные узкие резонансы с положением в широком диапазоне длин волн. Изменения параметра заполнения $\eta$ и толщины гетерослоя $h_{1}$ не дают такого эффекта. Моделирование оптических спектров проводилось как для вытянутых, так и сплюснутых эллипсоидов (с варьированием соотношения между осями). Оказалось, что вне зависимости от сплюснутости или вытянутости эллипсоидов (с одним и тем же отношением размеров их осей) расчетные спектры не изменяются. Это вполне объяснимо с точки зрения подхода Максвелла-Гарнетта, так как ориентация осей эллипсоидов случайна, и в среднем даёт одинаковый интегральный отклик - отклик гетерослоя. То есть слой по отношению к падающему излучению фактически содержит смесь из сплюснутых и вытянутых частиц.

Представленные выше результаты отражают закономерности лишь при углах падения $20^{\circ}$. Для получения более полной картины закономерностей в отражении ПМП на рис. 6 представлены угловые зависимости коэффициентов отражения для ППЗ и ППС для обоих состояний поляризации падающего света на основе экспериментальных и расчетных данных.

Данные расчетов соответствуют оптимально подобранным параметрам (как указано выше), при этом изменялся лишь угол падения света. Для ППЗ модель демонстрирует хорошее количественное совпадение с экспериментальными угловыми зависимостями (рис. 6, $a$ ). Зависимости для ППС (рис. 6,b), оставаясь качественно схожими для обоих состояний поляризации света, при $s$-поляризованном излучении демонстрируют некоторое количественное различие при больших углах падения [39]. Необходимо отметить, что, несмотря на 
незначительные количественные несоответствия, модель приемлемо описывает угловые закономерности в отражении света ПМП (особенно для ППЗ).

Как известно, возбуждение поверхностных плазмонов (поверхностных плазмон-поляритонов - ППП) на гладкой поверхности металла возможно, например, с использованием $p$-поляризованного излучения в конфигурации Кречмана, и невозможно при непосредственном падении излучения при любой поляризации. Однако возбуждая ППП, можно при определенных условиях получить эффект переизлучения падающей энергии в виде фотонов. Возбудить ППП возможно также при использовании шероховатых металлических поверхностей (как с регулярным, так и нерегулярным их распределением по поверхности). ПМП как раз и являются системой с нерегулярными наномасштабными шероховатостями. Так как такие пленки - это металлические наночастицы, находящиеся на сплошной металлической пленке, то очевидно, что условия возбуждения ППП с их использованием реализуемы. Обращает на себя внимание гипсохромный сдвиг максимума плазмонной полосы при $p$-поляризованном свете, начиная с углов падения $40^{\circ}$ и выше (рис. 4). При $s$-поляризованном свете ни при каких углах падения такого эффекта не наблюдается. Указанный сдвиг начинает проявляться лишь при больших углах падения, при которых волновой вектор падающего излучения равен или больше волнового вектора ППП. Расчетные спектры отражают указанную закономерность, что также может свидетельствовать о применимости используемой в работе модели, чувствительной к условиям возбуждения ППП.

\section{Заключение}

Модель, основанная на задаче о плоскослоистой структуре с включенным гетерослоем эффективной среды в приближении Максвелла-Гарнетта, показала свою пригодность к описанию отражательных свойств ППЗ и ППС. Продемонстрировано, что наличие слоя эффективной среды в расчетах приводит к формированию одной плазмонной полосы для ППЗ и двух плазмонных полос для ППС, как это регистрируется экспериментально. В отсутствие такого слоя плазмонные полосы в расчетном спектре отражения в принципе не проявляются. Модельные спектры качественно описывают все особенности в отражении света пленками, регистрируемые экспериментально. Для положения максимумов плазмонных полос и коэффициентов отражения в них модель дает к тому же количественное совпадение.

Разработанная в настоящей работе модель может быть обобщена на случай присутствия в гетерогенном слое наноэллипсоидов различных форм (с различным соотношением между полуосями), поскольку для этого случая имеются аналитические выражения для эффективной диэлектрической проницаемости [26]. Кроме того, модель можно расширить путем рассмотрения нескольких гетерослоев, с размещением в каждом из которых наноэллипсоидов заданной формы. Такие дополнения могут позволить достичь наилучшего согласия расчетных и экспериментальных результатов формированием широкого профиля плазмонных полос набором из более узких резонансов.

\section{Финансирование работы}

Исследование выполнено в рамках заданий 1.2.02 ГПНИ „Фотоника, опто- и микроэлектроника“ и 3.03 ГПНИ „Конвергенция - 2020“ при финансовой поддержке Министерства образования Республики Беларусь.

\section{Конфликт интересов}

Авторы заявляют, что у них нет конфликта интересов.

\section{Список литературы}

[1] Gaponenko S.V. // Phys. Rev. B. 2002. V. 65. N 14. P. 140303. doi 10.1103/PhysRevB.65.140303

[2] Kneipp K. // Phys. Today. 2007. V. 60. P. 40. doi 10.1063/1.2812122

[3] Stockman M.I. // Phys. Today. 2011. V. 64. P. 39. doi 10.1063/1.3554315

[4] Juan M.L., Righini M., Quidant R. // Nat. Photonics. 2011. V. 5. P. 349. doi 10.1038/nphoton.2011.56

[5] Leuthold J., Hoessbacher C., Muehlbrandt S., Melikyan A., Kohl M., Koos C., Freude W., Dolores-Calzadilla V., Smit M., Suarez I., Martínez-Pastor J., Fitrakis E.P., Tomkos I. // Opt. Photonics News. 2013. V. 24. P. 28. doi 10.1364/OPN.24.5.000028

[6] Tittl A., Giessen H., Liu N. // Nanophotonics. 2014. V. 3. P. 157. doi 10.1515/nanoph-2014-0002

[7] Ndukaife J.C., Kildishev A.V., Nnanna A.G.A., Shalaev V.M., Wereley S.T., Boltasseva A. // Nat. Nanotechnol. 2015. V. 11. P. 53. doi $10.1038 /$ nnano.2015.248

[8] Fong N.R., Berini P., Tait R.N. // Nanoscale. 2016. V. 8. P. 4284. doi 10.1039/C5NR08001K

[9] Петров Ю.И. Кластеры и малые частицы. М.: Наука, 1986. $368 \mathrm{c}$.

[10] Heilmann A. Polymer Films with Embedded Metal Nanoparticles. Berlin, Heidelberg: Springer-Verlag, 2003. $218 \mathrm{p}$.

[11] Markel V.A. // J. Opt. Soc. Am. A. 2016. V. 33. N 7. P. 1244. doi 10.1364/JOSAA.33.001244

[12] Maxwell Garnett J.C. // Philos. T. R. Soc. A. 1904. V. 203. P. 385. doi 10.1098/rsta.1904.0024

[13] Wei H., Eilers H. // J. Phys. Chem. Solids. 2009. V. 70. P. 459. doi 10.1016/j.jpcs.2008.11.012

[14] Optical Characterization of Real Surfaces and Films: Advances in Research and Development / Ed. by Vedam K., Francombe M.H., Vossen J.L. London: Academic Press, 1994. $328 \mathrm{p}$.

[15] Vieaud J., Merchiers O., Rajaoarivelo M., Warenghem M., Borensztein Y., Ponsinet V., Aradian A. // Thin Solid Films. 2016. V. 603. P. 452. doi 10.1016/j.tsf.2016.02.022 
[16] Gao X.-Y., Feng H.-L., Ma J.-M., Zhang Z.-Y., Lu J.-X., Chen Y.-S., Yang S.-E. Gu J.-H. // Physica B. 2010. V. 405. P. 1922. doi 10.1016/j.physb.2010.01.076

[17] Zhang Y., Zhou X., Cao K., Chen X., Deng Z., Liu S., Shan B., Chen R. // Thin Solid Films. 2015. V. 593. P. 144. doi 10.1016/j.tsf.2015.09.056

[18] Czajkowski K.M., Switlik D., Langhammer C., Antosiewicz T.J. // Plasmonics. 2018. V. 13. P. 2423. doi 10.1007/s11468-018-0769-4

[19] Morales-Luna G., Morales-Luna M. // Sci. Rep. 2020. V. 10. P. 5841. doi 10.1038/s41598-020-62706-4

[20] Pedrueza E., Valdés J.L., Chirvony V., Abargues R., Hernández-Saz J., Herrera M., Molina S.I., MartínezPastor J.P. // Adv. Func. Mater. 2011. V. 21. P. 3502. doi 10.1002/adfm. 201101020

[21] Feofanov A., Ianoul A., Kryukov E., Maskevich S., Vasiliuk G., Kivach L., Nabiev I. // Anal. Chem. 1997. V. 69. P. 3731. doi 10.1021/ac970304c

[22] Герман А.Е., Гачко Г.А. // Веснік ГрДУ імя Янкі Купалы. Сер. 2. Матэматыка. Фізіка. Інфарматыка, вылічальная тэхніка і кіраванне. 2001. № 2. С. 70.

[23] Vasilyuk G., Maskevich S., Sveklo I. // Abstr. 3rd Int. Symposium on Advanced Infrared and Raman Spectroscopy, Vienna (Austria), July 5-9, 1998. Vienna: Vienna University of Technology, 1998. P. 75.

[24] Kong J.A. Electromagnetic Wave Theory. NY.: John Wiley \& Sons, 1986. $667 \mathrm{p}$.

[25] Chew W.C. Waves and Fields in Inhomogeneous Media. NY.: IEEE Press, 1995. 608 p.

[26] Sihvola A.H., Kong J.A. // IEEE Trans. Geos. Remote Sens. 1988. V. 26. N 4. P. 420. doi $10.1109 / 36.3045$

[27] Niklasson G.A., Granqvist C.G., Huderi O. // Appl. Opt. 1981. V. 20. N 1. P. 26. doi 10.1364/AO.20.000026

[28] Wangberg R., Elser J., Narimanov E.E., Podolskiy V.A. // J. Opt. Soc. Am. B. 2006. V. 23. N 3. P. 498. doi 10.1364/JOSAB.23.000498

[29] Toal B., McMillen M., Murphy A., Hendren W., Atkinson R., Pollard R. // Mat. Res. Lett. 2014. V. 1. P. 015801 (1-11). doi 10.1088/2053-1591/1/1/015801

[30] Zhang X., Wu Y. // Sci. Rep. 2015. V. 5. P. 7892 (1-7). doi 10.1038/srep07892

[31] Ораевский А.Н., Проценко И.Е. // Письма в ЖЭТФ. 2000. T. 72(9). С. 641.

[32] Ораевский А.Н., Проченко И.Е. // Квант. электрон. 2001. T. 31. № 3. C. 252.

[33] Anguelova M.D. // J. Geophys. Res. 2008. V. 113. P. C08001 (1-22). doi 10.1029/2007JC004212

[34] Liu S.-B., Wei E.-B., Ding X. // J. Appl. Remote Sens. 2013. V. 7. P. 073598 (1-12). doi 10.1117/1.JRS.7.073598

[35] Pierson J.F., Rousselot C. // Surf. Coat. Technol. 2005. V. 200. P. 276. doi 10.1016/j.surfcoat.2005.02.005

[36] Van Duyne R.P., Hulteen J.C., Treichel D.A. // J. Chem. Phys. 1993. V. 99. P. 2101. doi $10.1063 / 1.465276$

[37] Johnson P.B., Christy R.W. // Phys. Rev. B. 1972. V. 6. P. 4370. doi 10.1103/PhysRevB.6.4370

[38] Noguez C.J. // J. Phys. Chem. C. 2007. V. 111. P. 3806. doi $10.1021 / \mathrm{jp} 066539 \mathrm{~m}$

[39] Борн М., Вольф Э. Основы оптики. М.: Наука, 1973. 719 с. 